\title{
An MRI Image Analysis of Primary Cardiac Neoplasms
}

\author{
Ya-Nan Jin \\ Jing-Liang Cheng (10) \\ Yan Zhang \\ Xiao-Ning Shao \\ Xiao-Pan Zhang \\ Wen-Bo Zhang
}

Department of Magnetic Resonance Imaging, First Affiliated Hospital of Zhengzhou University, Zhengzhou, 450052, People's Republic of China
Correspondence: Jing-Liang Cheng Department of Magnetic Resonance Imaging, First Affiliated Hospital of

Zhengzhou University, Zhengzhou, 450052, People's Republic of China

Tel $+86 \quad 13603863860$

Fax +8637166271510

Email jingliangcheng123@163.com
Objective: This study aimed to examine the magnetic resonance imaging (MRI) characteristics of primary cardiac neoplastic lesions.

Methods: A retrospective investigation was conducted on 24 cases of primary cardiac neoplastic lesions as confirmed by surgery and pathology results. All the cases in this study received MRI multi-sequence and multi-dimension scanning, including the cardiac long-axis and short-axis cine sequences, parameter sequences of the cardiac long axis and short axis (T1WI, T2WI), first-pass perfusion sequence, and delayed enhancement sequence of the cardiac long axis and short axis. The age and gender of the patients and the location, size, signal characteristics, and relationship with the neighboring tissues of all the lesions were examined.

Results: Twenty-four cases of primary neoplastic lesions were examined in this study, the onset age was 11-72 years old, the median age was 53 years old, and the mean age was 46 years old. Among these cases, there were 8 cases including males and 16 cases including females, 19 cases were benign lesions; including 11 cases of myxoma, 4 cases of hemangioma, 1 case of paraganglioma, 1 case of PEcoma, 1 case of hamartoma, and 1 case of lipoma. The malignant lesions included 3 sarcomas and 2 lymphomas in 5 patients.

Conclusion: MRI imaging provides a great value in the preoperative classification of primary cardiac neoplastic lesions.

Keywords: cardiac neoplasms, magnetic resonance imaging, comparative study

With the rising recognition of cardiac magnetic resonance imaging (CMRI) scanning, more primary cardiac neoplastic lesions have been established, diagnosed, and treated during the early stages of the disease. ${ }^{1}$ CMRI can be used to detect the structure and function of the heart and the association between the tumor tissue and the wall, the tumor tissue and the cavity of the heart, and pericardium. The clinical indications of primary cardiac neoplastic disease lack specificity and are complex and varied while there is no precise laboratory index for this. ${ }^{2,3}$ The MRI data for this disease are few and preoperative classifications are challenging. In this study, 24 cases of primary cardiac tumors were collected and the clinical and MRI characteristics were examined to improve the accurateness of a preoperative diagnosis of cardiac neoplastic disease.

\section{Information and Methods Clinical Data}

From January 2011 to June 2019, 24 patients with benign and malignant cardiac tumors that were established by pathology testing were enrolled in this study from 
the First Affiliated Hospital of Zhengzhou University. The patients enrolled in this study included 8 males and 16 females. The patients' age ranged within 11-72-years-old, the median age was 53-years-old, and the average age was 46-years-old. Nineteen patients had benign tumors, including 11 cases of myxomas, 4 cases of hemangiomas, 1 case of paraganglioma, 1 case of PEComa, 1 case of hamartoma, and 1 case of lipoma. Five patients had malignant tumors, including 3 cases of sarcomas and 2 cases of lymphoma. The main clinical symptoms included chest pain while some patients had no clear symptoms that were discovered by a physical examination. All the patients' diagnoses were confirmed by operation and pathology within 2 weeks.

\section{Equipment and Methods}

The Siemens Skyra 3.0T MRI scanner, 18 channel surface phased array coil, and MRI compatible with Peru electrocardiogram gating were used for the scanning in this study. The scan methods were as follows: (1) The cardiac long axis and short axis cine sequences; (2) The parameters of the cardiac long axis and short axis (T1WI, T2WI); (3) The perfusion sequence of the cardiac long axis and short axis; and (4) The delayed enhancement sequence (1 patient did not undergo this scanning); First-pass perfusion and delayed enhancement sequence. The contrast agent, GdDTPA, was used. A high-pressure syringe was used for the bolus injection in the patients' elbow vein; the flow rate was $3 \mathrm{~mL} / \mathrm{s}$ and the dose was $0.2 \mathrm{mmol} / \mathrm{kg}$. Subsequently, $20 \mathrm{~mL}$ of normal saline was injected and delayed enhancement scanning was performed 10 minutes after administering the injection with the contrast agent.

\section{Results}

\section{The Benign Heart Lesions}

Myxoma was found in 11 patients, of which myxoma was found in the left atrium in 3 patients, in the right atrium in 4 patients, in the right ventricle in 3 patients, and the left ventricle in 1 patient. Amongst these patients, 6 were female and 5 were male. The MRI manifestations of myxoma are as follows: The lesions had diverse sizes and the longest diameter was $1.6-7.7 \mathrm{~cm}$. Most of the lesions were polypoid and lobulated and demonstrated heterogeneous signal shadows while the long diameters were mainly significantly greater than the short diameters. T1W1 presents isointense or slightly hyper-intensive signals (when compared with normal myocardium). In a small proportion of patients, it may present slightly hyper-intensive signals. In the present study, T1W1 presented slightly hyper-intensive signals in 1 patient and T2W1 presented apparent homogenous or heterogenous hyper-intensive signals. The heart cine sequence of MRI presented hypo-intensive signals, the first-pass perfusion mostly presented hypo-intensive signals, and slightly hyper-intensive signals were evident on the margins of certain lesions. The delay enhanced scan revealed that the central part of the lesion presented hypo-intensive signals, the margins of the lesions were slightly enhanced, and the enhancement of the tumor pedicle was evident in lesions with the tumor pedicle. In the present study, nearly all of the patients had this delayed enhancement characteristic; only 1 patient presented clear enhancement in the delayed phase and the lesion was small, with an approximate diameter of $1.6 \mathrm{~cm}$. In the present study, the heart function of 3 patients was slightly reduced, the lowest ejection fraction was $47 \%$, the lesion had a diameter of $7.7 \mathrm{~cm}$, which was the largest lesion, the lesion was located in the right ventricle, and grew across the tricuspid valve. In the other 2 patients, the lesions were located in the right atrium and it was evident that the lesions moved across the tricuspid valve in the cardiac cycle. Myxoma of the heart is largely differentiated according to thrombus; the thrombi have irregular shapes, low activity and no pedicle, and present hypo-intensive signals in each sequence (Figure 1).

Hemangioma was found in 4 patients. Amongst these patients, hemangioma was found in the left ventricle in 1 patient, in the left atrium in 1 patient, in the outflow tract of the right ventricle in 1 patient, and in the left supraventricular pericardium in 1 patient. Amongst these patients, 3 were female and 1 was male. The diameter of the lesions was within $1.3-7.3 \mathrm{~cm}$, the biggest lesion was in the pericardium; the pathological type was cavernous hemangioma, T1W1 presented isointense, slightly hypo-intensive or slightly hyper-intensive signals and T2WI presented hyper-intensive or slightly hyper-intensive signals. The delay enhanced scan revealed that the lesions demonstrated aclearenhancement, some signals were not homogenous, and hypo-intensive signal shadows were evident in the largest lesion (Figure 2).

Paraganglioma was found in 1 patient. The patient was a young woman and the lesion was located at the pericardium of the top of the left atrium, had a diameter of approximately $7.7 \mathrm{~cm}$, was quasi-round, and had welldefined margins. Paraganglioma of the heart presented 


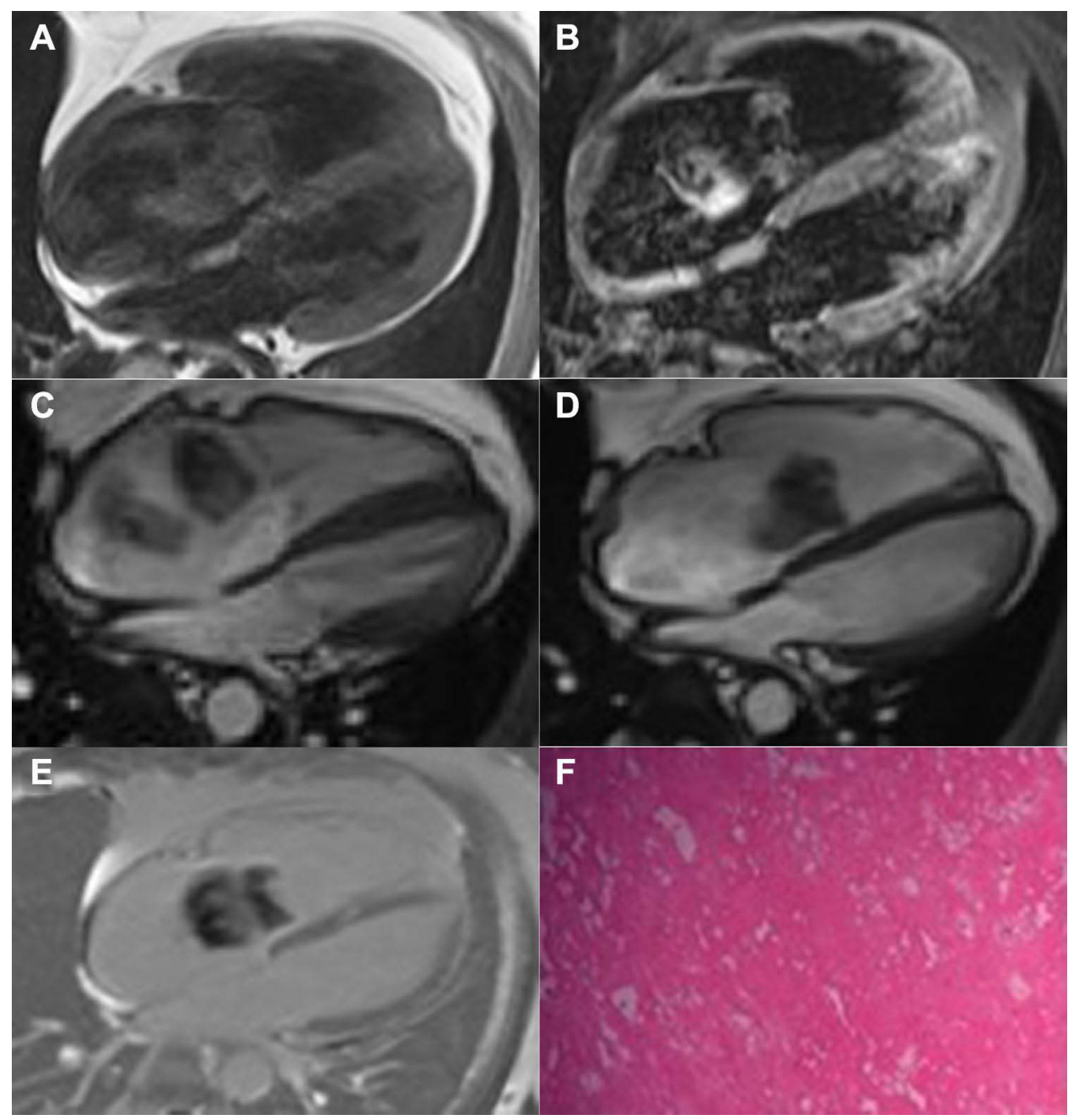

Figure I A 30-year-old male patient, the lesion in the right atrial. (A) the lesion presented isointense signals on TIWI (four-chamber heart); (B) the lesion presented hyperintensive signals fat-suppressed T2WI (four-chamber heart); (C) Four-chamber cine sequence of the ventricular systolic phase presented right atrial space-occupying; (D) During diastolic phase, the four-chamber cine presented that the right atrium mass entered the right ventricle with blood flow; (E) The delayed enhanced four-chamber sequence presented inhomogeneous enhancement on the margin of the lesion; $(\mathbf{F})$ The pathological findings indicated myxoma $(\mathrm{H} \& \mathrm{E}, \times 200)$.

isointense signals on T1WI and hyper-intensive signals on T2W1, strip flow empty vascular shadows were also evident around the lesion, in the early stage of the first-pass perfusion cardiac cycle, a clear enhancement of most parts of the tumor could be seen and at a later stage, the degree of enhancement was decreased. The delay enhanced scan revealed that the tumor demonstrated mild heterogenous enhancement. The blood supply of the paraganglioma of the heart mainly came from the direct or indirect branches of the coronary artery and disordered vascular masses were rare.

PEcoma was found in 1 patient; the patient was female. The lesion was located in the pericardium behind the left ventricle and below the left atrium, had a diameter of approximately $4.6 \mathrm{~cm}$, was quasi-round, and had well-defined margins. The MRI imaging findings are as follows: T1W1 showed isointense or slightly hypo-intensive signals, pointed or flaky slightly hyper-intensive signals are evident in it and T2W1 showed isointense or slightly hyper-intensive signals and pointed or flaky higher signals are evident in it. The delay enhanced scan revealed that the lesions showed slightly hypointensive signals and strip and flake enhanced shadows were evident at the margins and in the center of the lesion.

Hamartoma was found in 1 patient; the patient was male. The lesion was located in the inferior wall of the left ventricle, which had a diameter of approximately $2.8 \mathrm{~cm}$ and had relatively well-defined margins, but the margins were not smooth. T1W1 presented hyperintensive signals, T2W1 presented hyper-intensive signals but the signals of the lesions were not homogenous and strip and flaky hypo-intensive signals were evident on the T1W1 and T2W1 images. 


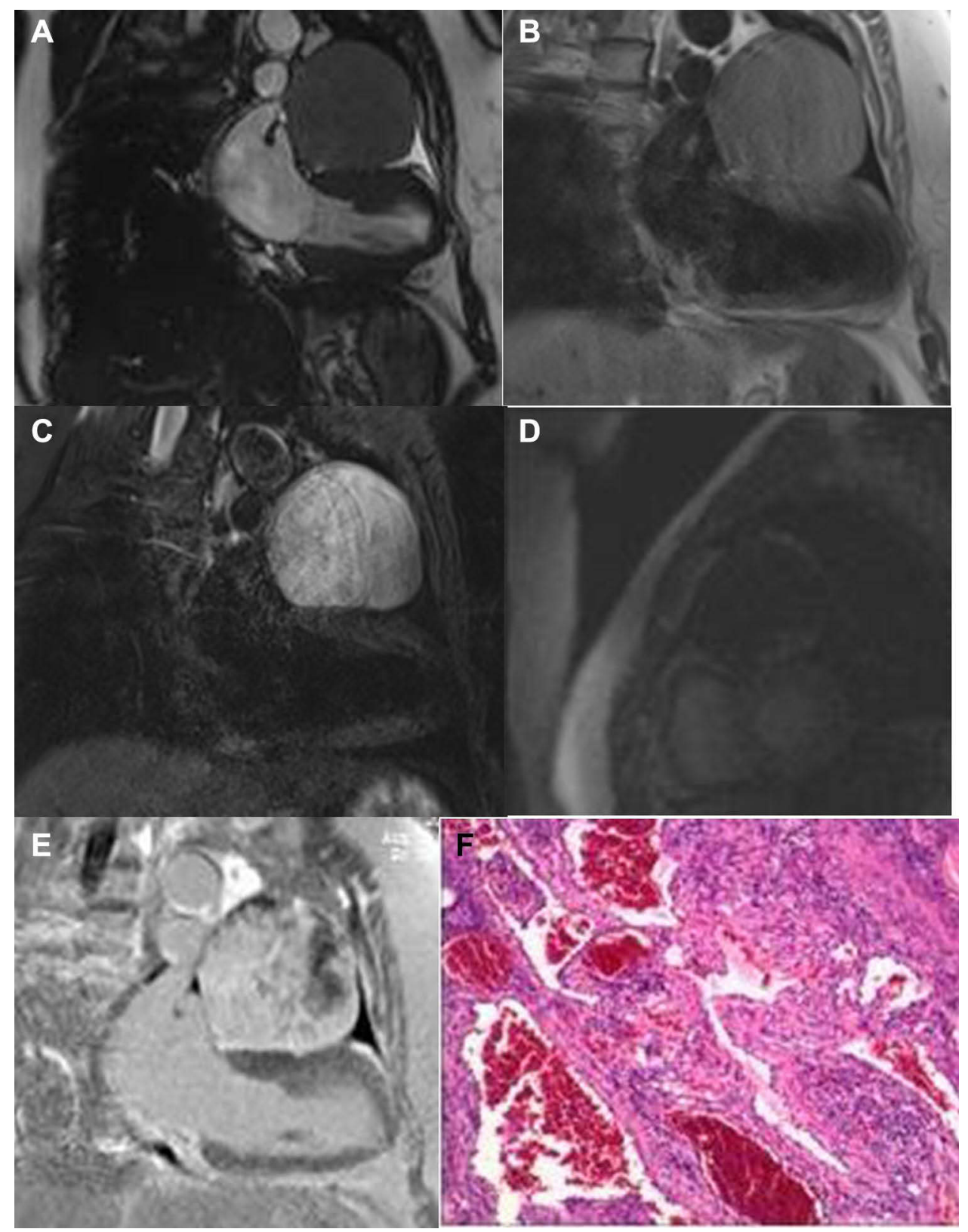

Figure 2 A 60-year-old female patient, a pericardial space-occupying region on the upper part of the left ventricle. (A) Two-chamber cine sequence presented hypointensive signals on the space-occupying in the upper left ventricle, the margins were clear and the signals were homogenous; (B) TIWI (two-chamber heart) presented isointense or slightly hyper-intensive signals in lesions; (C) T2WI presented hyper-intensive signals; (D) First-pass perfusion presented peripheral enhancement; (E) The delayed enhanced sequence presented inhomogeneous enhancement on the margins of the lesion; and the hypo-intensive signal area around the lesion; (F) The pathological findings indicated cavernous hemangioma (H\&E, × 200).

Lipoma was found in 1 patient; the patient was female. The lesion was located in the right atrium, which had a diameter of approximately $8.5 \mathrm{~cm}$, had well-defined margins, and the signals were homogenous. T1W1 and T2W1 presented hyper-intensive signals.

\section{Malignant Heart Lesions}

Sarcoma was found in 3 patients. Amongst these patients, sarcoma was found in the blood vessels in 2 patients; 1 of them had epithelioid hemangioendothelioma and in the other patient, the sarcomas were located on the top and lateral wall of the right atrium. In the 3rd patient, the sarcomas were found in the superior vena cava and the right atrium. Synoviosarcoma was found in 1 patient, which were on the left atrioventricular site and superior pericardium, had diameters ranging within $3.5-11.9 \mathrm{~cm}$, the angiosarcoma and synovial sarcoma had indistinct margins, and 
demonstrated heterogenous signals. The MRI imaging findings were as follows: $\mathrm{T} 1 \mathrm{~W} 1$ presented isointense or hypo-intensive signals, patchy hyper-intensive signals were evident in it and T2W1 presented isointense signals, patchy and hyper-intensive signals were evident in it. Fat-suppressed imaging revealed that the lesions presented slightly hyper-intensive signals, the short T1 long T2 signal presented hyper-intensive signals, the lesions presented heterogenous enhancement in the first-pass perfusion, the delay enhanced scan revealed that clear heterogenous enhancement could be seen in the parenchyma of lesions, and the short T1 and long T2 image revealed that there was no enhancement in the center of the lesion (Figure 3).

Lymphomas were found in 2 patients; both patients were female. The lymphomas had diameters of 5.5$7.4 \mathrm{~cm}$ and the lesions were all located in the right atrium and had an indistinct boundary with the right atrial wall. The MRI imaging findings were as follows: T1W1 presented isointense or slightly hyper-intensive signals, T2W1 presented hyper-intensive signals, the tumor was attached to the right atrial wall with a wide base, part of the right atrial wall was involved and thickened, heterogenous and slightly hyper-intensive signals were evident

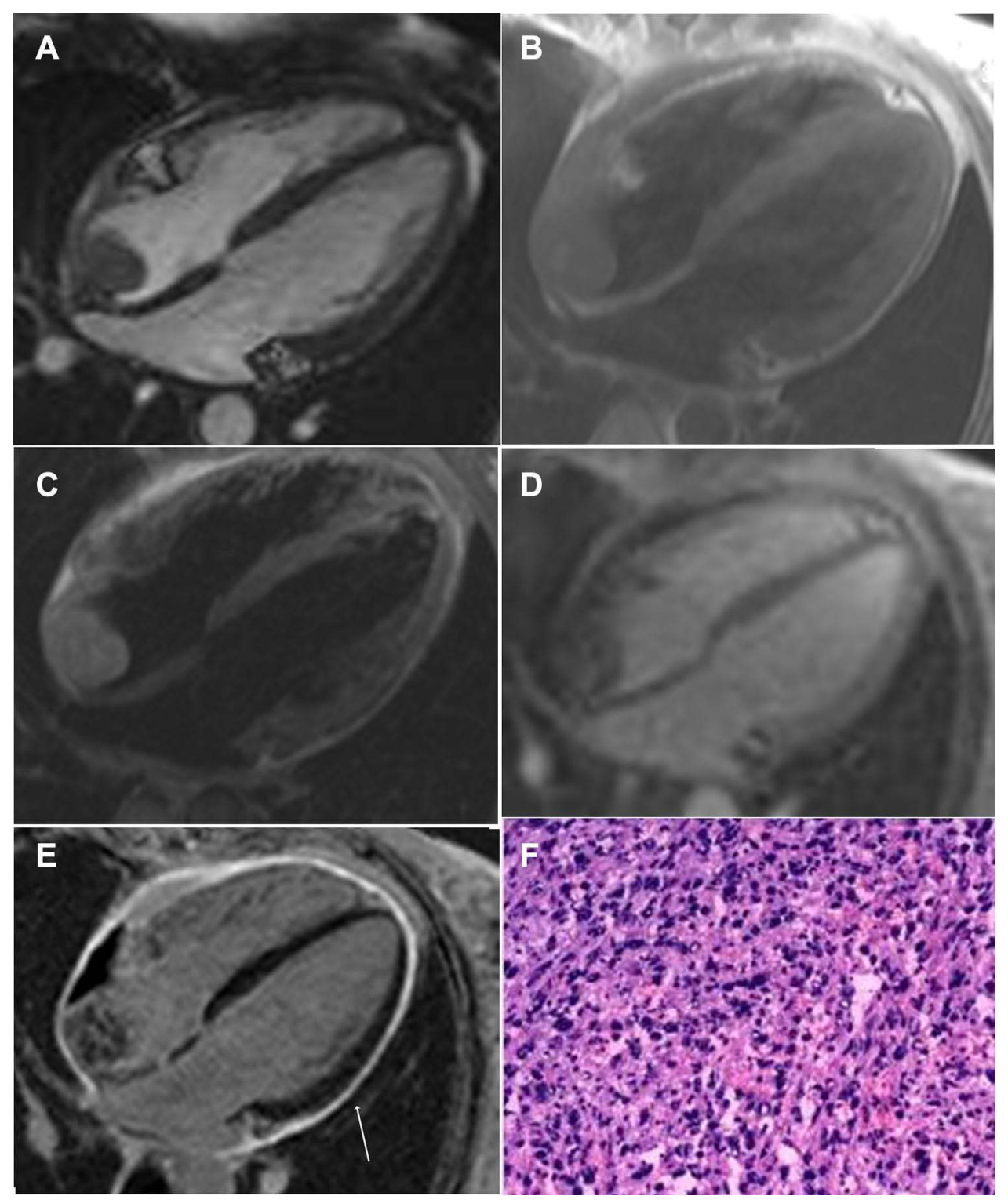

Figure 3 A 34-year-old female patient. (A) Four-chamber heart cine sequence presented hypo-intensive signals in the space-occupying the right atrium; (B) TIWI presented wide-basedisointense or slightly hyper-intensive signals in the right atrium; (C) Fat-suppressed T2WI presented slightly hyper-intensive signals in the lesion; (D) The first-pass perfusion presented slightly inhomogeneous enhancement of the right atrial space; (E) The delayed enhanced sequence presented inhomogeneous enhancement on the margins of the lesion; (F) The pathological findings indicated angiosarcoma (H\&E, $\times 200)$. 
during the first-pass perfusion, the delay enhanced scan revealed that heterogenous enhancement were evident in certain lesions, and some lesions presented hypo-intensive signals without clear enhancement (Figure 4).

\section{Discussion}

Cardiac myxoma is the most common benign tumor of the heart, which can appear in both the atrial and ventricular cavities. This tumor mainly occurred in the left atrium (approximately 75\%) followed by the right atrium (approximately 20\%) and rarely occurred in the ventricles (approximately 5\%). ${ }^{4}$ In the present study, the incidence of tumors in the left and right atrium was the same and the incidence of tumors in the ventricle was $33.3 \%$, these results are different from previous reports and the incidence was not unusual. The disease often occurs in females and the age of onset is typically 30-60-yearsold. In the present study, the age of onset was 16-72years-old and the range of the age of onset was more widespread. A previous study showed that the enhancement mode of myxoma was gradual enhancement from the tumor pedicle to the center because of the radial distribution of the blood vessels from the tumor pedicle to the center. ${ }^{5}$ But, Liu et al reported that the delay enhanced scan of myxoma showed the tumor pedicles were visibly enhanced. ${ }^{6}$ The present study, however, showed that the
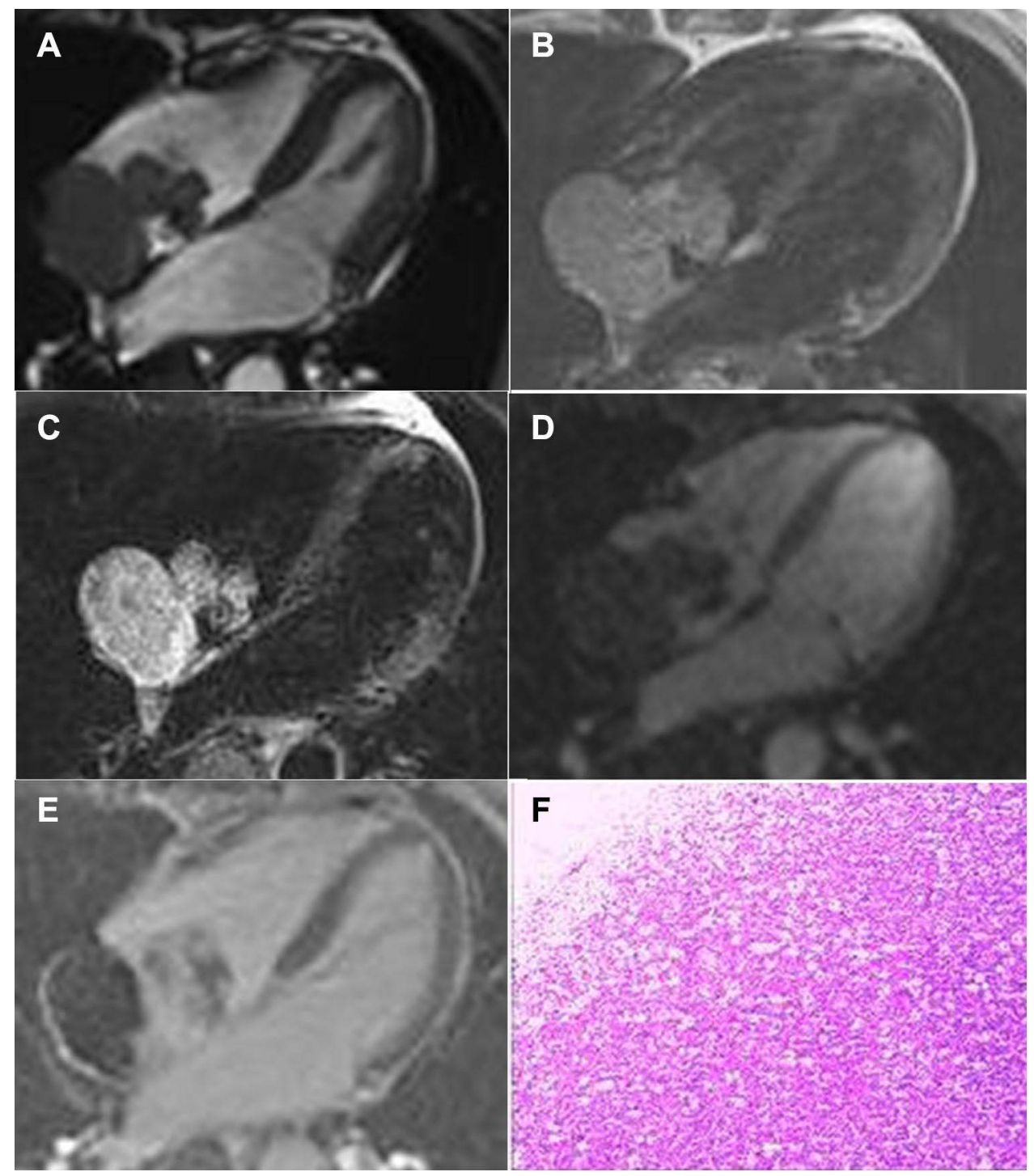

Figure 4 A 55-year-old female patient, right atrial space-occupying region. (A) Four-chamber heart cine sequence presented hypo-intensive signals in the space-occupying region in the right atrium; (B) TIWI presented slightly hyper-intensive signals; (C) T2WI presented heterogenous hyper-intensive signals; (D) The enhancement of the firstpass perfusion was not clear; (E) The delayed enhancement showed that part of the lesion presented inhomogeneous enhancement; $(\mathbf{F})$ The pathological findings indicated lymphoma $(\mathrm{H} \& \mathrm{E}, \times 200)$. 
enhancement mode of myxoma was mostly gradual enhancement from the tumor pedicle to the center, but the degree of enhancement was only slight. The enhancement was only visible in 1 patient and the lesion was located in the right ventricle. The reasons for this result may be as follows: The lesions were small, in which there were many components of fibrous tissue and blood vessels. When some myxomas are large and move across the valve, it is easy to affect the cardiac function.

Cardiac hemangioma is a rare primary tumor of the heart and can be divided into cavernous hemangioma, capillary hemangioma, and tendril hemangioma according to the histopathological types. The incidence of cavernous hemangioma is relatively high. ${ }^{7,8}$ In the present study, cavernous hemangioma was found in 2 patients and capillary hemangioma was found in 1 patient. There was no significant difference in the onset age and gender for this disease.

Paraganglioma is a rare neuroendocrine tumor, which is even more unusual in the heart, and primarily occurs in the left atrial visceral ganglion, therefore, it is more often located at the top or posterior wall of the left atrium and more common in women than men ${ }^{9}$ and in young adults. The case in the study was consistent with previous literature. ${ }^{10} \mathrm{~A}$ non-enhanced scan of this case revealed that the strip flow empty vascular shadows were evident around the tumor. This characteristic could also be seen in paraneuromas in other sites, ${ }^{11}$ which is a characteristic imaging manifestation of paraganglioma and the vessel may be a blood supply artery or a blood stasis vein. ${ }^{12}$ The presence of metastasis is a reliable basis for the diagnosis of benign and malignant paraganglioma. In this patient, no metastasis was observed by positron emission tomography-computed tomography (PET-CT).

PEcoma is also known as a perivascular epithelioid cell differentiation tumor and is a kind of mesenchymal tumor with the features of perivascular epithelioid cells and can occur in all parts of the body, including the kidneys, lungs, uterus, pancreas, rectum. PEcoma in the heart is fairly rare. ${ }^{13}$ PEcoma often occurs in young and middle-aged women, most cases include benign lesions, and the lesions usually show round or quasi-round mass shadows. Previous studies on PEcoma in the liver revealed that the lesions presented hypo-intensive signals and hyperintensive signals on $\mathrm{T} 2 \mathrm{~W} 1$, the delay enhanced scan revealed that the lesions presented hypo-intensive signals, and punctate or linear vascular shadows and delayed enhancement of the pseudo capsule could also be seen. ${ }^{14,15}$ The manifestation of the lesions, in this case, was similar to that in previous literature.

Hamartoma of the heart is very rare; patients typically have no clear clinical symptoms and the lesions are found by physical examinations. Hamartoma of the heart grows slowly, is a benign lesion, and its base is as hard as bone attaching to the heart. When the tumor is large, it can affect the vascular functions and cause corresponding signs or symptoms. In this case of hamartoma, both T1WI and T2WI showed endocardial mass high signals; this suggested that the content of fat in it was high and flaky low signals were evident around it. The delay enhanced scan revealed that there was no clear enhancement in the tumor, but enhancement was evident around the tumor.

A cardiac lipoma is a rare primary tumor of the heart, which can occur at any age and the common sites of incidence include the right atrium, left ventricular subendocardium, and pericardium. ${ }^{16}$ There are two types of cardiac lipomas: Solitary and infiltrative lipomas. Solitary cardiac lipomas usually have clear margins and a complete envelope, but infiltrative cardiac lipomas have unclear margins and the tumor can infiltrate the myocardium. ${ }^{17}$ The lesions, in this case, were solitary lipoma, the lesion was located in the right atrium, and the diameter was approximately $8.5 \mathrm{~cm}$. The heart cine sequence of MRI revealed that the lesions showed high signals, low signal capsular shadows were evident around the tumor mass, and the margins were well-defined. The MRI images of the lesions showed typical homogenous hyper-intensive signals on T1W1 and hyper-intensive signals on T2W1.

Malignant primary tumors of the heart are also relatively rare, accounting for $10-25 \%$ of tumors of the heart, $75 \%$ of which are cardiac sarcoma. Among them, angiosarcoma is the most common, followed by undifferentiated sarcoma, osteosarcoma, leiomyosarcoma, and synoviosarcoma. In this study, 2 patients had angiosarcoma, 1 patient had synoviosarcoma, and 1 of the angiosarcomas was low malignant epithelioid hemangioendothelioma. Angiosarcoma is also called malignant hemangioendothelioma and is a malignant tumor originating from the vascular endothelial cells. Primary angiosarcoma of the heart usually occurs between the age of 30-60-years-old, the average age is 39years-old. This tumor typically occurs in males, the tumor masses are usually found in the right atrium and epicardium, and the proportion of the lesions in the right atrium is as high as $93 \% .^{18,19}$ The findings in the present study was 
consistent with previous studies; the tumor occurred in the right atrium in 1 patient and occurred at the top of the right atrium and under the epicardium of the lateral wall in another patient, the wall of the right atrium was affected and visibly thickened, there was no clear specificity in the MRI image, T1W1 presented isointense or slightly hypointensive signals, T2W1 presented heterogenous hyperintensive signals, hypo-intensive signals were evident in it, strip and flaky slight enhancement could be seen in the firstpass perfusion, and delay enhanced scan revealed clear heterogenous enhancement. In 1 patient with angiosarcoma, pericardial thickening was evident and the delay enhanced scan revealed that the lesions presented clear enhancement. This patient was considered to have pericarditis.

Synoviosarcoma is a kind of malignant tumor with the characteristics of epithelial or mesenchymal biphasic differentiation. Case reports in previous studies revealed that the onset age of cardiac synoviosarcoma was 16-64-yearsold, this tumor occurred more frequently in males and usually occurred in the pericardial cavity. ${ }^{20}$ This case of synoviosarcoma was also found in the pericardium of the left atrioventricular sidewall, which manifested as a massshaped mixed-signal shadow, the boundary with the pericardium was not clear, and the left atrioventricular septum showed pressure changes. The researcher also examined that the center of the lesion may have been bleeding. Previous studies on MRI analysis of cardiac synoviosarcoma are rare. A previous study in China reported that T1W1 presented isointense signals, T2W1 presented "Triple signal" signs, that is, isointense, hypo-intensive, slightly hyper-intensive or obviously hyper-intensive signals. The cases in this study were consistent with the literature and the characteristic indications were also observed.

Primary cardiac lymphomas are very rare, accounting for $1.3 \%$ of primary cardiac tumors and most of them are B-cell lymphoma, which usually affects the right heart; the most common onset site is the right atrium. There was no significant difference in the age and gender of the patients with this disease. In the present study, both cases of cardiac lymphoma were B-cell lymphoma and both lesions were located in the right atrium, these were consistent with the findings shown in previous literature. Cardiac lymphoma has indistinct margins, irregular shapes, and poor activity. $^{21,22}$

The limitation of this study is that there were few cases and only conventional sequences were used to summarize the appearances of cardiac tumor lesions. In the future, more cases should be collected for subsequent study and quantitative analyses of tumor lesions should be completed using conventional and updated sequences to better diagnose or differentially diagnose cardiac tumors.

\section{Conclusions}

In summary, the MRI characteristics of primary cardiac tumors have certain features and these features combined with the disease history can improve the accurateness of preoperative diagnoses.

\section{Ethics Statement}

This study was conducted with approval from the Ethics Committee of First Affiliated Hospital of Zhengzhou University. This study was conducted in accordance with the declaration of Helsinki. Written informed consent was obtained from all participants. A parent or legal guardian of patients under the age of 18 years provided informed consent.

\section{Disclosure}

The authors report no conflicts of interest in this work.

\section{References}

1. Kumar P, Singh A, Deshmukh A, Kumar S. Cardiac MRI for the evaluation of cardiac neoplasms. Clin Radiol. 2020;75(4):241-253.

2. Zerbo S, Argo A, Maresi E, Liotta R, Procaccianti P. Sudden death in adolescence caused by cardiac haemangioma. J Forensic Leg Med. 2009;16(3):156-158. doi:10.1016/j.jflm.2008.08.020

3. Tzani A, Doulamis IP, Mylonas KS, Avgerinos DV, Nasioudis D. Cardiac tumors in pediatric patients: asystematic review. World J Pediatr Congenit Heart Surg. 2017;8(5):624-632. doi:10.1177/ 2150135117723904

4. Rahmanian PB, Castillo JG, Sanz J, Adams DH, Filsoufi F. Cardiac myxoma: preoperative diagnosis using a multimodal imaging approach and surgical outcome in a large contemporary series. Interact Cardiovasc Thorac Surg. 2007;6(4):479-483. doi:10.1510/ icvts.2007.154096

5. Deetjen A, Möllmann S, Dill T, Hamm CW. Left atrial myxoma diagnosed and characterized by cardiac magnetic resonance imaging. $Z$ Kardiol. 2005;94(10):695. doi:10.1007/s00392-0050278-2

6. Liu Y, Kong X-Q, Wang Z-P, Xu H-B, Liu D-X, Kong X-C. Study on MRI and surgical pathology of cardiac mucus tumor. Chin J Radiol. 2009;43(10):1043.

7. Wang M-Y, Gao C-Q, Xiao C-S, Wu Y, Wang Y, Zhang L. 4 cases of cardiovascular tumor. Chin J Thorac Cardiovasc Surg. 2011;27 (3): 182 .

8. Kipfer B, Englberger L, Stauffer E, Carrel T. Rare presentation of cardiac hemangiomas. Ann Thorac Surg. 2000;70(3):977-979. doi:10.1016/S0003-4975(00)01666-0

9. Walker T, Bail DH, Schmid E, Haeberle L, Kuehne H, Ziemer G. Challenging the advanced: cardiac surgery without awareness of a pheochromocytoma. Thorac Cardiovasc Surg. 2006;54(7):498-499. doi:10.1055/s-2006-924096

10. Zhu M-H, Deng Y-B, Liu Y-N. 1 case of primary and side neuroblastoma of the heart after the diagnosis of cardiomyopathy and postoperative follow-up. Chin J Ultrason. 2009;18(12):1092-1093. 
11. Yuan J, Pei-yi G. MRI diagnosis of secondary neuroblastoma in the vertebral tube. J Pract Radiol. 2015;31:352.

12. Gelabert-González M, Pita-Buezas L, Arán-Echabe E. Paraganglioma of the cauda equina. Korean J Spine. 2015;12(1):29. doi:10.14245/ kjs.2015.12.1.29

13. Musella A, De Felice F, Kyriacou AK, et al. Perivascular epithelioid cell neoplasm (PEComa) of the uterus: a systematic review. Int J Surg. 2015;19:1-5. doi:10.1016/j.ijsu.2015.05.002

14. Liu J-Z, Xie H, Si-bin L. 6 cases of multi-layer helix CT performance in 6 cases of epithelial cytoma in the liver's blood vessels. Chin $J$ Med Imaging. 2012;09:41.

15. Lee SJ, Kim SY, Kim KW, et al. Hepatic angiomyolipoma with minimal fat, mimicking hepatocellular carcinoma. Clin Mol Hepatol. 2012;18(3):330-335. doi:10.3350/cmh.2012.18.3.330

16. Miller DV, Tazelaar HD. Cardiovascular pseudoneoplasms. Arch Pathol Lab Med. 2010;134(3):362-368. doi:10.5858/134.3.362

17. Amano J, Nakayama J, Yoshimura Y, Ikeda U. Clinical classification of cardiovascular tumors and tumor-like lesions, and its incidences. Gen Thorac Cardiovasc Surg. 2013;61(8):435-447. doi:10.1007/ s11748-013-0214-8
18. Combes M, Rottey S, Backer TD. Cardiovascular toxicity of cancer treatment. Acta Clin Belg. 2011;66(4):260. doi:10.2143/ACB.66. 4.2062566

19. Zhang Z-Y, Gao X, Bai C-M, Zhang S-Y, Qi M. Clinical imaging features and prognosis analysis of 16 cases of primary hemangiosarcoma of the heart. Chin J Cardiol. 2019;47(9):731.

20. Xu Y-F, Hou Y-Y, Luan L-J. Clinical pathological characteristics of heart slip sarcoma. Chin J Clin Med. 2019;26(01):86-89.

21. Jonavicius K, Salcius K, Meskauskas R, Valeviciene N, Tarutis V, Sirvydis V. Primary cardiac lymphoma: two cases and a review of literature. J Cardiothorac Surg. 2015;10:138. doi:10.1186/s13019-0150348-0

22. Yin J, Hua S-H, Li L-J. 1 case of primary heart lymphoma. Chin $J$ Med Imaging Technol. 2016;32(6):880.
International Journal of General Medicine

\section{Publish your work in this journal}

The International Journal of General Medicine is an international, peer-reviewed open-access journal that focuses on general and internal medicine, pathogenesis, epidemiology, diagnosis, monitoring and treatment protocols. The journal is characterized by the rapid reporting of reviews, original research and clinical studies
Dovepress

across all disease areas. The manuscript management system is completely online and includes a very quick and fair peer-review system, which is all easy to use. Visit http://www.dovepress.com/ testimonials.php to read real quotes from published authors. 